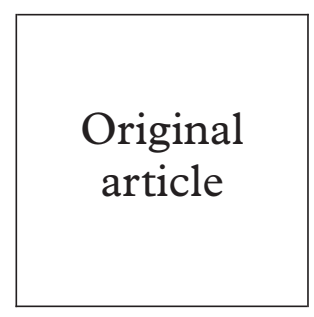

Original article

Sexually Transmitted Infections Research Centre, University of Sydney, Australia C S Estcourt

C Marks

A M Johnson

A Mindel

Sydney Sexual Health Centre, Sydney Hospital, GPO Box 1614, Sydney 2001

R Rohrsheim

B Donovan

Department of Public Health and Community Medicine, University of Sydney, Australia

C S Estcourt

B Donovan

A Mindel

Correspondence to: Dr Claudia Estcourt Claudia.Escourt@royalhos-tr. nthames.nhs.uk

Accepted for publication 20 April 2000

\title{
HIV, sexually transmitted infections, and risk behaviours in male commercial sex workers in Sydney
}

Claudia S Estcourt, Caron Marks, Richard Rohrsheim, Anne M. Johnson, Basil Donovan, Adrian Mindel

Objectives: To assess prevalence of HIV and sexually transmitted infections (STIs), risk behaviours, and demographics in male commercial sex workers (CSWs)/prostitutes in Sydney.

Methods: Retrospective, cross sectional study with two comparison groups. Demographic, behavioural, and morbidity data were analysed from standardised medical records of patients attending a public STI and HIV service in Sydney between January 1991 and March 1998. Two comparison groups were used: female CSWs and non-CSW working homosexual men who attended over the same time.

Results: 94 male CSWs, 1671 female CSWs, and 3541 non-CSW working homosexual men were included. The prevalence of HIV in male CSWs tested (6.5\%) was significantly greater than in female CSWs $(0.4 \%, \mathrm{p}=0.0001)$, but less than in non-CSW homosexual men $(23.9 \%$, $\mathrm{p}<0.0001)$. Genital warts occurred significantly more frequently in male CSWs than in comparison groups. Prevalence of other STIs was similar in all groups. Male CSWs saw significantly fewer clients per week than female CSWs and male and female CSWs used condoms with almost all clients. Male CSWs reported significantly more non-work sexual partners than female CSWs and non-CSW homosexual men and were significantly more likely to have unprotected penetrative sex with their non-work partners than non-CSW homosexual men. Injecting drug use was significantly more frequent in male CSWs than in both comparison groups.

Conclusions: Although male CSWs use condoms with clients, they are more likely to practise unsafe sex with non-work partners (especially women) and inject drugs than female CSWs and non-CSW homosexual men. Some men with HIV are working within the commercial sex industry. Targeted health education to encourage safer drug use and safer sex outside work is needed. (Sex Transm Inf 2000;76:294-298)

Keywords: male commercial sex workers; prostitutes; HIV; sexually transmitted infections

\section{Introduction}

Men and women who work in the commercial sex industry differ from the general population by their increased vulnerability to infection, their potential for increased rates of sexually transmitted infections (STIs) and blood borne virus (BBV) infections, and their specific needs in terms of health care. ${ }^{1}$ It is possible that within any society a subset of commercial sex workers (CSWs), with large numbers of unprotected sexual partners, could act as a core group of disease transmitters. ${ }^{1}$ Their clients may then transmit infection to sexual partners outside the sex industry and to other sex workers. The extent of this sexual mixing will affect the spread of STIs and BBVs in the general population. A sound understanding of the characteristics of commercial sex workers and the nature of sex work will help to guide development of appropriate healthcare services for this group and ultimately may lead to decreased rates of infection in the community as a whole.

The limited studies of men who exchange money for sex suggest that they may have high rates of STIs including human immunodeficiency virus (HIV).$^{2-4}$ However, few studies have addressed sexual behaviour, partner choices, and patterns of infection. Previous reports have tended to concentrate on one group of male CSWs, defined by the type of work undertaken - for example, street work or escort roles. This has led to a limited understanding of the spectrum of experiences across the industry and a lack of appreciation of potential differences in healthcare needs within the male CSW population. ${ }^{5}$

In order to investigate the hypotheses that male CSWs have increased prevalences of STIs and HIV and that male CSWs' private lives place them at increased risk, we conducted a study to investigate the characteristics of male CSWs who attended an inner city sexual health service with respect to prevalence of STIs and BBVs, sexes and numbers of sexual partners, demographics, condom usage with clients and other partners, type of sex work undertaken, and drug use.

\section{Materials and methods}

CSWs (prostitutes) were defined as men and women who reported currently exchanging sex for money or other services. CSWs were identified from the clinic database either by self classification on the clinic patient registration form or in response to a specific question about participation in commercial sex work as part of the routine medical history.

Cases were all male CSWs who first attended the Sydney Sexual Health Centre, a public STI 
and HIV service in the central business district of the city, between January 1991 and March 1998. Two comparison groups were used: all female CSWs and all non-CSW homosexual men who first attended over the study period. As so little is known about male CSWs, there was no readily identifiable group with which to compare them and it was unclear which variables it was appropriate to match for. To address these issues we chose two comparison groups from other clinic attenders: female CSW and non-CSW homosexual men. This enabled comparison with another societal group in which sex is sold, and comparison with a group of men whose sexual partners better reflected the sex of sexual partners chosen by the majority of the male CSW. Variables such as age and numbers of sexual partners were all explanatory variables of interest, which might explain the different STI epidemiology between the groups. For this reason, no matching was considered appropriate.

For the purposes of this study, non-CSW homosexual men were defined as men who reported exclusively male sexual partners in the 12 months immediately before registration and denied sex work. Non-CSW homosexual men were chosen as a comparison group because the majority ( 51 of $92,55 \%$ ) of male CSWs reported male non-work partners in the 12 months before presentation.

All new attenders at SSHC were offered a comprehensive sexual health screen to include: Gram staining and culture from appropriate sites for Neisseria gonorrhoeae, testing for Chlamydia trachomatis (by cell culture from 1991 to 1995 and from on then by PCR), routine Gram stain of high vaginal secretions, and wet preparation for Trichomonas vaginalis in women. Genital herpes was diagnosed clinically and/or by culture. Genital warts were diagnosed clinically. In addition, serological testing for hepatitis B, HIV, and syphilis were offered to all patients. From 1992, patients who reported risk behaviour for hepatitis $\mathrm{C}$ acquisition were offered serological testing and men who reported sex with men were offered serological testing for hepatitis A.

Data were recorded at the time of presentation using a standardised medical record form and then entered onto the clinic database.

Analysis was performed retrospectively. Continuous variables such as age were treated as categorical variables by grouping. Comparisons were made between male CSWs and female CSWs and male CSWs and non-CSW homosexual men using $\chi^{2}$ tests for categorical variables, and Student's $t$ test, Fisher's exact, and Mann-Whitney tests as appropriate. Statistical analysis was performed using the sPsS package.

The study was approved by South Eastern Sydney Area Health Service research ethics committee.

\section{Results}

DEMOGRAPHIC VARIABLES

Ninety four male CSWs, 1671 female CSWs, and 3541 non-CSW homosexual men first attended the clinic during the 1991-8 study period and were included in the study. Male CSWs were similar in age to female CSWs (median ages 25.5 years, range $17-59$ and 26.0 years, range 16-63, respectively) but were significantly younger than the non-CSW homosexual men (median age 30.0 years, range $14-83, p<0.0001)$. Just over half $(49,55.1 \%)$ of the male CSW were born in Australia. This was similar to the non-CSW homosexual men $(1998,56.6 \%)$ but significantly greater than the female CSWs $(580,35.6 \%, \mathrm{p}<0.0001)$, of whom $749(46.0 \%)$ were born in south or South East Asia.

Area of permanent residence was similar across the groups although data were missing for $39-43 \%$ of variables as identifying information such as name and address was optional for all patients. The vast majority of respondents resided in the Sydney metropolitan area: male CSWs 44/50 (88.0\%); female CSWs 796/ 875 (91.0\%); non-CSW homosexual men $1659 / 2033(81.6 \%)$. Few of the male CSWs were permanently resident overseas (5/50, $10 \%$ ) compared with $158 / 873(18.1 \%)$ of the female CSWs but this was not statistically significant.

\section{PREVALENCE OF STIS}

Prevalence of individual STIs was broadly similar across the groups with the exception of anogenital warts (table 1): $20.9 \%$ of the male CSWs were found to have warts at presentation and this was the commonest STI diagnosis. The prevalence of warts was significantly higher in male CSWs than in both female CSWs $(4.6 \%, \mathrm{p}<0.0001)$ and non-CSW homosexual men $(12.2 \%, \mathrm{p}=0.017)$. Gonorrhoea was diagnosed in three male CSWs ( $4.7 \%$ of those tested) which included one case of anorectal gonorrhoea, and in 152 (7.4\% of those tested) non-CSW homosexual men, which included 46 cases of anorectal gonorrhoea.

Almost one third of the male CSWs were diagnosed as having one or more of gonorrhoea, chlamydia, syphilis, genital warts, and genital herpes at first attendance compared with around $15 \%$ of female CSWs ( $\mathrm{p}=$ 0.0001 ) and $20 \%$ of non-CSW homosexual men $(\mathrm{p}<0.0001)$. Multiple STI diagnoses were uncommon in all groups (table 1 ).

Very few participants were tested for hepatitis $\mathrm{C}$ in spite of a history of injecting drug use. Half of the female CSW who were tested and one third of tested non-CSW homosexual men were found to be hepatitis C antibody positive. Only three male CSW were tested and none was positive (table 1).

Significantly more male CSWs were HIV positive (four, $6.5 \%$ of those tested) than female CSWs (four, $0.4 \%$ of those tested, $p$ $<0.0001$ ) and these men were all aware of their HIV status at presentation. Two of the HIV positive male CSWs reported injecting drug use. All of the HIV positive male CSWs had exclusively male sexual partners out of work. Two of the four HIV positive female CSWs were not previously known to be HIV positive at presentation. Significantly fewer male CSWs 
Table 1 Prevalence of STIs and BBVs in male CSWs, female CSWs, and homosexual men

\begin{tabular}{llll}
\hline & Male CSWs & Female CSWs & $\begin{array}{l}\text { Non-CSW homosexual } \\
\text { Men }\end{array}$ \\
\hline Number & $94^{\star}$ & $1671 \dagger$ & $3541 \ddagger$ \\
Gonorrhoea (all sites) & $3 / 64(4.7 \%)^{\star \star}$ & $40 / 1403(2.9 \%)$ & $152 / 2050(7.4 \%)$ \\
Chlamydia (all sites) & $1 / 58(1.7 \%)$ & $73 / 1370(5.3 \%)$ & $32 / 1700(1.9 \%)$ \\
Trichomoniasis & 0 & $6 / 1047(0.6 \%)$ & 0 \\
Syphilis (all stages) & $2 / 68(2.9 \%)$ & $21 / 1154(1.8 \%)$ & $45 / 2217(2.0 \%)$ \\
Genital warts & $19 / 91(20.9 \%)$ & $76 / 1660(4.6 \%)$ & $426 / 3502(12.2 \%)$ \\
Genital herpes (culture positive and clinical diagnosis) & $7 / 91(7.7 \%)$ & $69 / 1660(4.2 \%)$ & $158 / 3502(4.5 \%)$ \\
Hepatitis A (acute and chronic) & $0 / 17$ & $0 / 31$ & $11 / 655(1.7 \%)$ \\
Hepatitis B (acute and chronic) & $1 / 60(1.7 \%)$ & $24 / 1029(2.3 \%)$ & $44 / 1750(2.5 \%)$ \\
Hepatitis C (chronic) & $0 / 3$ & $26 / 51(51.0 \%)$ & $26 / 81(32.1 \%)$ \\
HIV & $4 / 62(6.5 \%)$ & $4 / 1112(0.4 \%)$ & $451 / 1885(23.9 \%)$ \\
No STI diagnosis & $61 / 91(67.0 \%)$ & $1366 / 1660(82.3 \%)$ & $2720 / 3502(77.7 \%)$ \\
One or more STIף & $29 / 91(31.9 \%)$ & $259 / 1660(15.6 \%)$ & $710 / 3502(20.3 \%)$ \\
Two STIsף & $1 / 91(1.1 \%)$ & $31 / 1660(1.9 \%)$ & $65 / 3502(1.9 \%)$ \\
Three or more STIs & $0 / 91$ & $4 / 1660(0.2 \%)$ & $7 / 3502(0.2 \%)$ \\
\hline
\end{tabular}

^Data missing for $3(3.2 \%)$ male CSW.

†Data missing for $11(0.7 \%)$ female CSW.

$\neq$ Data missing for $39(1.1 \%)$ homosexual men.

$\star \star$ Percentages refer to numbers positive of numbers tested.

ๆSTI = gonorrhoea, chlamydia, trichomoniasis, syphilis, genital warts, and genital herpes.

were HIV positive than the non-CSW homosexual men $(23.9 \%$ of those tested, $\mathrm{p}<0.0001)$.

However, there were significant differences in the proportions of each group tested for certain infections for the following comparisons: gonorrhoea (male CSW (68\%) versus female CSW (84\%), $\mathrm{p}=0.001$; male CSW versus nonCSW homosexual men (58\%), p=0.024), chlamydia (male CSW (62\%) versus female CSW (82\%), $\mathrm{p}=0.0001)$; male CSW versus non-CSW homosexual men $(48 \%), \mathrm{p}=0.005)$, syphilis (male CSW $(72 \%)$ versus non-CSW homosexual men $(63 \%), p=0.027)$, hepatitis $B$ (male CSW (63\%) versus non-CSW homosexual men $(49 \%), p=0.003)$, and HIV (male CSW (66\%) versus non-CSW homosexual men $(52 \%), \mathrm{p}=0.007)$.

A history of STIs was a common finding in all subjects: 37 of $94(40.2 \%)$ male CSWs, 612 of $1671(37 \%)$ female CSWs, and 1668 of $3541(47.2 \%)$ non-CSW homosexual men reported one or more of gonorrhoea, chlamydia, trichomonas, syphilis, genital warts, genital herpes in the past (differences not significant).

DRUG AND ALCOHOL USE

A history of injecting drug use was significantly more common in male CSWs $(17.4 \%)$ than in female CSWs $(9.1 \%, \mathrm{p}=0.016)$ and homosexual men $(7.6 \%, p=0.002)$. Of the 16 male CSWs who injected drugs, three reported exclusively female sexual partners out of work in the previous 12 months, seven reported exclusively male sexual partners and three reported both male and female sexual partners out of work. Excess alcohol intake, defined in this study as consumption of over $140 \mathrm{~g}$ per week for women and $280 \mathrm{~g}$ per week for men (the national recommended weekly maximum) was uncommon in all groups and was reported by $4.3 \%$ of the male CSWs, $7.4 \%$ of female CSWs, and $5.7 \%$ of the non-CSW homosexual men (differences not significant).

SEX WITH CLIENTS

Male CSWs reported significantly fewer clients per week (median six, range 1-32) than female CSWs (median 20, range 1-120) p <0.0001 (table 2$)$. Nine $(10 \%)$ of the male CSWs serv- iced both male and female clients and the remainder had an exclusively male clientele; only two female CSWs reported female clients. In all, $85.7 \%$ of male CSWs and $88.4 \%$ of female CSWs reported $100 \%$ condom usage for anal and/or vaginal sex in the previous 3 months.

SEX WITH NON-PAYING PARTNERS

In the preceding 12 months, outside of paid sexual encounters, 34 (37\%) reported exclusively male partners, $16(17 \%)$ reported male and female partners, 31 (34\%) reported exclusively female partners, and $11(12 \%)$ reported no non-commercial partners (data missing for two male CSWs). Male CSWs reported significantly more male and female partners in the past 12 months than female CSWs, $\mathrm{p}<0.0001$ (table 3). Male CSWs also reported significantly higher mean numbers of male nonpaying partners in the past 12 months than homosexual men, p $<0.0001$ (table 3 ). The range for numbers of sexual partners was very wide: 999 was the maximum number of partners which could be entered on the database and was reported by a very small number of participants in each group.

In contrast with client interactions, only around half of male and female CSWs reported consistent condom usage with their non-paying partners. Male CSWs were significantly less likely to report consistent condom usage with non-work partners than the non-CSW homosexual men, $p=0.002$ (table 3). However male CSWs who reported male non-commercial

Table 2 Characteristics of the sex worker-client relationship

\begin{tabular}{lll}
\hline & Male CSWs & Female CSWs \\
\hline $\begin{array}{l}\text { Number } \\
\text { Clients per week }\end{array}$ & $49 \dagger$ & $1030 \ddagger$ \\
$\quad$ mean (SE) & $9.3(1.7)$ & $24.0(0.6)$ \\
$\quad$ median (range) & $6.0(1-32)$ & $20(1-120)$ \\
Consistent condom usage & $28(85.7)$ & $793(88.4)$
\end{tabular}

*Data included from 17 October 1994, when these questions first appeared on the standardised medical records. tData missing for $47 \%$ of variables.

$\ddagger$ Data missing for $18 \%$ of variables.

\$100\% condom usage in past 3 months for vaginal and/or anal sex excluding condom breaks or slippage. 
Table 3 Characteristics of non-commercial sexual relationships

\begin{tabular}{|c|c|c|c|}
\hline & Male CSWs & Female CSWs & $\begin{array}{l}\text { Non CSW-homosexual } \\
\text { men }\end{array}$ \\
\hline Number $(\%)$ & $94 \dagger$ & $1671 \ddagger$ & 3541 \\
\hline \multicolumn{4}{|c|}{ Male and female non-paying partners in past 12 months } \\
\hline Mean (SE) & $42.2(12.9)$ & $32.5(3.4)$ & $14.8(0.71)$ \\
\hline Median (range) & $5(0-999)$ & $1(0-999)$ & $5(1-999)$ \\
\hline \multicolumn{4}{|c|}{ Male non-paying partners in past 12 months } \\
\hline Mean (SE) & $36.2(12.1)$ & $32.3(3.4)$ & $14.8(0.71)$ \\
\hline Median (range) & $1(0-999)$ & $1(0-999)$ & $5(1-999)$ \\
\hline Consistent condom usage & $37(49.3)$ & $625(49.4)$ & $1668(66.5)$ \\
\hline
\end{tabular}

†Data missing for $2(2.1 \%)$ male CSWs

$\neq$ Data missing for $20-29 \%$ of variables.

$\llbracket 100 \%$ condom usage in past 3 months for vaginal and/or anal sex excluding condom breaks or slippage.

partners were significantly more likely to use condoms consistently $(27 / 51,54 \%)$ than male CSWs with exclusively female non-commercial partners $(6 / 31,19 \%, \mathrm{p}=0.01)$.

\section{Discussion}

Almost one third of male CSWs who attended our sexual health service had one or more STIs at clinical presentation. This was double the prevalence of STIs in female CSWs and significantly greater than the STI prevalence in non-CSW homosexual men. These results support published observations from a number of different countries which report increased rates of STIs in male CSWs compared with the general population. ${ }^{246-8}$ However, genital warts accounted for most of the difference in STI prevalence and was the commonest single STI diagnosis. We acknowledge that the significant differences observed between the comparison groups in the proportions of participants tested for certain infections may have provided a source of bias.

The seroprevalence of HIV in the male CSWs was around $6.5 \%$ and was considerably lower than the seroprevalence in non-CSW homosexual men and, consistent with observations in Sydney almost a decade ago. ${ }^{9}$ In contrast, studies from other countries report considerably higher prevalence of HIV in male CSWs of $13-27 \% .^{2-4} 1011$ However, sampling bias may have contributed to the differences in reported prevalence as this study was conducted in a clinical setting and many of the men were seeking HIV care. An age effect also may have contributed as the male CSWs were significantly younger than the non-CSW homosexual men. In addition, although male CSWs were less likely to use condoms consistently with non-work partners than the nonCSW homosexual men, further analysis of the data showed that it was the male CSWs with exclusively female non-work partners who were more likely to have unprotected sex than the male CSWs who reported male partners. Nevertheless, male CSWs were significantly more likely to be HIV positive than female CSWs and the few HIV infections among female CSWs reflect infections imported from South East Asia. ${ }^{12}$ Although the numbers were small, the finding that half the HIV positive male commercial sex workers had also injected drugs may have important consequences for spread of infection into other societal groups.

Almost $20 \%$ of the male CSWs in our study gave a history of injecting drug use. Substance use may contribute to acquisition of STIs and BBVs directly by the exchange of infectious body fluids or by influencing judgment and decision making abilities. The latter may result in unsafe sexual practices and consequent risk of sexually acquired infection. ${ }^{6}$ Over one third of the male CSWs who injected drugs reported female non-commercial sexual partners. This suggests a further route of spread of BBVs and STIs into the heterosexual community. This result is in keeping with Pleak and MeyerBahlburg's 1990 observations of high rates of injecting drug use in male sex workers in New York. ${ }^{13}$

In spite of the high rates of injecting drug use reported in male CSWs in this study, the prevalences of hepatitis B and C were low. However, less than one fifth of male CSW who had a history of injecting drug use were tested for antibody to hepatitis C. Further interpretation of these data is limited by the retrospective nature of the study. Information on injecting frequency, substances used and sharing of equipment was not routinely collected and testing for hepatitis $C$ was not available in the early part of the study. One Australian study of men who injected drugs and had homosexual contact reported that injecting practices were generally safe. ${ }^{14}$ Further work is required to understand the injecting practices and context of injecting in our male CSW population.

Male CSWs saw far fewer clients per week than female CSWs. This may reflect the differences in nature and context of male and female sex work or may be due to sampling bias. In Estep et al's study, ${ }^{6}$ the number of clients serviced per night was associated with the type and location of the sex work. Male street workers reported two to three times the number of clients than escort or independent commercial sex workers. Information on location of sex work and the type of sexual practice undertaken was not routinely recorded in our study. Male street workers in Sydney tend to operate out of the eastern part of the city and may access local sexual health services in preference to our own.

All the male CSWs in this study reported male clients and 10\% also reported servicing female clients. This finding is in keeping with earlier observations on male and trans-sexual prostitution in Australia. ${ }^{15}$ More information on the nature of the sexual services undertaken with female clients is needed. None the less, it is clear that this could be one route of spread of STIs and BBVs from male commercial sex work into the heterosexual community. ${ }^{8}$

Reported condom use within commercial sexual relationships was high for both male and female CSWs. However, instances of unprotected high risk sex did occur and it is possible that it is the clients from encounters in which safer sex is not successfully negotiated who pose the greatest risk to the sex worker. ${ }^{5}{ }^{16}$ Self reported condom use may be unreliable and given that participants were questioned in a sexual health clinic, and the desire to give "the right answer" to the doctor may have falsely elevated estimates of usage. 
In common with previous studies, ${ }^{3}$ our data suggest that male CSWs' non-commercial sexual behaviour may render them at greater risk of acquiring and transmitting infection than sex with clients. Male CSWs reported significantly more non-paying sexual partners than female CSWs and non-CSW homosexual men. However, one third of male CSWs reported exclusively female sexual partners and $11 \%$ had no non-commercial partners in the preceding 12 months. Although consistent condom usage for all male CSWs with non-paying partners was significantly less than that reported by non-CSW homosexual men, it was the male CSW with exclusively female partners who reported more unprotected sex. Data on the type of sex undertaken are not available in this study but previous work suggests that anal sex is more common with non-commercial partners than with clients. ${ }^{6}$

We assume some selection bias in our study as we relied on a sample of clinic attenders, who would be more likely to be symptomatic and who may not adequately represent all types of male CSWs in Sydney.

This study supports observations from other countries that the prevalence of STIs and HIV in male CSWs is a cause for concern. Although condom usage with clients appears to be high, male CSWs were more likely to practise unsafe sex with non-commercial partners and inject drugs than female CSWs and non-CSW homosexual men. Their vulnerability to infection and their potential for disease transmission appear to be more related to non-commercial sex and risk behaviours, than paid sexual encounters. Historically, stigmatisation and marginalisation have created barriers for men working within the industry to accessing the health care and services they need. Our data argue strongly for the need for appropriate health services, targeted education, and support for behaviour change, especially in relation to sexual behaviour out of work.
Contributors: CSE had the original idea for the study, study design, data analysis, and interpretation, and writing of the manuscript; CM performed data analysis and interpretation, and review of manuscript; RR performed data retrieval and analysis; AMJ was involved with study design and review of manuscript; BD performed study design and review of manuscript; AM was involved with study design, data interpretation, and review of manuscript.

Conflicts of interest: Nil.

The authors are very grateful to Dr Robin Tideman for assistance with data analysis and interpretation, and to $\mathrm{Dr}$ Richard Hillman for his helpful review of the manuscript.

1 Day S, Ward H. Sex workers and the control of sexually transmitted disease. Genitourin Med 1997;73:161-8.

2 Coutinho RA, van Andel RLM, Rijsdijk TJ. Role of male prostitutes in spread of sexually transmitted diseases and human immunodeficiency virus. Genitourin Med 1988;64: 207-8.

3 Tomlinson DR, Hillman RJ, Harris JRW, et al. Screening for sexually transmitted disease in London-based male prostitutes. Genitourin Med 1991;67:103-6.

4 Kunawararak P, Beyrer C, Natpratan C, et al. The epidemiology of HIV and syphilis among male commercial sex workers in Northern Thailand. AIDS 1995;9:517-21.

5 Browne J, Minichiello, V. The social meaning behind male sex work: implications for sexual interactions. $\mathrm{Br} F$ Sociol 1995;46:598-622.

6 Estep R, Waldorf D, Marotta T. Sexual behavior of male prostitutes. In: Huber J, Schneider BE, eds. The social con109.

7 Simon PM, Morse EV, Balson PM, et al. Barriers to human immunodeficiency virus related risk reduction among male street prostitutes. Health Ed Q 1993;20:261-73.

8 McKeganey NP. Prostitution and HIV: what do we know and where might research be targeted in the future? AIDS 1994;8:1215-26.

9 Griggs L, Alan D. Male sex workers and HIV in Sydney: a "potted history". Nat AIDS Bull 1990:20-1.

10 Morse EV, Simon PJ, Osofsky HJ, et al. The male street prostitute: a vector for transmission of HIV infection into the heterosexual world. Soc Sci Med 1991;32:535-9.

11 Elifson KW, Boles J, Sweat M. Risk factors associated with HIV infection among male prostitutes. Am $\mathcal{F}$ Public Health 1993;83:79-83.

12 O'Connor CC, Berry G, Rohrsheim R, et al. Sexual health and use of condoms among local and international sex workers in Sydney. Genitourin Med 1996;72:47-51.

13 Pleak RR, Beyer-Bahlburg HFL. Sexual behaviour and AIDS knowledge of young male prostitutes in Manhattan. f Sex Res 1990;27:557-87.

14 Crofts N, Marcus L, Meade J, et al. Determinants of HIV risk among men who have homosexual sex and inject drugs. AIDS Care 1995;7:647-55.

15 Prestage G, Crawford J, Van de Ven P, et al. Sydney Men and Sexual Health. Report Series C: Report C. 2: Sexual identity and sexual behaviour with both men and women in a sample of homosexually-active men in Sydney, Australia. sample of homosexually-active men in Sydney, Aus

16 Bloor MJ, Barnard MA, Finlay A, et al. HIV-related risk practices among Glasgow male prostitutes: reframing concepts of risk behaviour. Med Anthrop $Q$ 1993;7:152-69. 